Revue européenne des sciences sociales

European Journal of Social Sciences

XLIV-134 | 2006

Quel(s) défi(s) pour les sciences sociales à l'heure de la mondialisation?

\title{
L'information déniée
}

Michele Cangiani

\section{(2) OpenEdition}

\section{Journals}

Édition électronique

URL : http://journals.openedition.org/ress/286

DOI : $10.4000 /$ ress.286

ISSN : 1663-4446

Éditeur

Librairie Droz

\section{Édition imprimée}

Date de publication : 1 juin 2006

Pagination : 81-95

ISBN : 9-782-600-01095-5

ISSN : 0048-8046

Référence électronique

Michele Cangiani, «L'information déniée », Revue européenne des sciences sociales [En ligne],

XLIV-134 | 2006, mis en ligne le 14 octobre 2009, consulté le 21 avril 2019. URL : http://

journals.openedition.org/ress/286; DOI : 10.4000/ress.286 


\section{Michele CANGIANI}

\section{L'INFORMATION DÉNIÉE}

R. Lynd publia en 1939 son essai sur la méthode et le rôle des sciences sociales dans une société composée d'individus, dynamique, technologiquement et administrativement développée, dont il analyse la complexité croissante comme «l'allongement des chaînes de causalité » (Lynd 1964: p. 212). Une telle société a la capacité d'aménager une quantité croissante d'information; elle en a aussi la nécessité, pour contrecarrer le désordre, l'entropie.

Les pages qui suivent concernent quelques réponses apportées par la science économique au défi de la complexité moderne, et donc à la question de l'information dans son sens le plus général. L'économie «globalisée» ne pourrait exister, dit-on, sans le support des technologies de l'information et de la communication. Mais le problème de l'information ne peut être limité à cette seule donnée technologique: il concerne avant tout la qualité, le sens et les tendances du système économique mondial. Comme tout système, celui-ci est défini par son organisation, et donc par l'information qu'il sélectionne et élabore, par le rapport qu'il entretient avec son environnement.

Les théories aussi sont des systèmes. Je voudrais proposer quelques arguments en faveur de la pensée «institutionnaliste», qu'on pourrait appeler «classique» pour la distinguer, dans le domaine des théories économiques, des théories néoclassiques comme de celles néo-institutionnalistes. Le paradigme institutionnaliste est toujours resté minoritaire, et a souvent été ignoré ou mal compris, bien qu'il soit plus complexe et donc plus à même de rendre significative une quantité beaucoup plus vaste d'informations que les théories «normales», et de soulever des problèmes qui sinon resteraient invisibles.

\section{MONOPOLES ET MONOCULTURES}

Il peut être utile, en introduction, de rappeler certaines manifestations de la tendance existant actuellement, dans notre «société de l'information» et «de la connaissance», à rendre inutilisable ou impossible une information qui serait utile, voire nécessaire; et à conditionner ou limiter la production et la diffusion de la connaissance.

La création d'un vaste domaine - une «toile» mondiale - où l'information puisse être librement et gratuitement échangée, diffusée et augmentée, comme instrument de la démocratie aussi, se heurte à des exigences politiques et économiques. Par exemple, les deux phases du Sommet mondial sur la société de l'information (à Genève en décembre 2003 et à Tunis en novembre 2005) ont révélé 
l'importance géopolitique du contrôle de la gestion d'Internet, à laquelle les EtatsUnis refusent de renoncer en faveur de l'ONU. D'autre part, les grandes entreprises s'intéressent de plus en plus aux activités dans lesquelles la connaissance est fondamentale comme facteur productif et comme produit. Dans des cas semblables, il apparaît d'autant plus vrai que la connaissance garantit un avantage compétitif et par conséquent des profits, à condition que le monopole en soit assuré sur une période assez longue.

La législation sur les droits d'auteur et les brevets a changé à partir des années 1980, et ce changement est parti des Etats-Unis. La protection a été étendue dans le temps, qui va maintenant jusqu'à 75 ans, et à de nouveaux domaines, des logiciels et des programmes d'ordinateur à la recherche sur le vivant. Les conditions de la brevetabilité - les critères d'utilité, de nouveauté et de spécificité de l'invention - sont devenues moins sévères. Les universités ont été incitées à stipuler des accords avec les entreprises privées, de préférence nationales, pour l'exploitation des résultats de leurs recherches, même si celles-ci ont bénéficié de fonds publics (comme prévu par la Loi Bayh-Dole, 1980). Les brevets ont commencé ainsi à être étendus à la recherche fondamentale et à devenir de véritables «droits d'exploration» dans plusieurs champs du savoir; la distinction entre invention (technologique) et découverte (de ce qui existe dans la nature) s'avère donc compromise. (Voir p. ex. Azam 2005, Coriat 2002, et d'autres articles dans les mêmes numéros des deux revues.)

La production intellectuelle risque d'être transformée progressivement en propriété privée et finalisée au profit; avec pour résultat que le progrès de la connaissance se trouve non seulement orienté dans des directions qui ne sont pas nécessairement les plus utiles, mais aussi ralenti. Le caractère cumulatif du développement devient plus difficile et plus coûteux. "La prolifération des brevets en amont des innovations, sur une connaissance fractionnée dans le champ de la recherche fondamentale - observe G. Azam (2005 : p. 24) -, compromet les innovations en l'aval.»

Un caractère particulier de la connaissance est qu'elle peut être partagée et utilisée sans limites, sans se réduire, sans devenir plus rare; au contraire, plus elle est utilisée et partagée, plus elle augmente. Mais la médiation de l'organisation sociale - de l'organisation dominante dans notre temps - intervient comme un filtre informatif pour donner à la connaissance une forme sociale spécifique: celle d'une marchandise. Elle devient un bien «économique» dans le sens qui est celui de cette forme sociale: avoir une valeur pour le marché, être produite et échangée avec profit. Il faut, à cette fin, la rendre institutionnellement rare; sans cela, comme l'enseigne la science économique reconnue, elle n'aurait pas de «valeur».

Plusieurs chercheurs, groupes et mouvements s'opposent à la «marchandisation de la connaissance» et en particulier de celle qui concerne la nature vivante. On évoque parfois des raisons éthiques à ce propos; mais il y a aussi une raison très pratique: en soumettant la connaissance à la logique instrumentale et réductionniste du marché et du profit, on risque de rendre impossible l'adaptation «coévolutive» de la société à son environnement humain et naturel, et donc l'existence même du genre humain.

Je dois me limiter à rappeler un seul exemple à ce propos, les recherches de V. Shiva qui montrent le caractère contre-adaptif de ce qu'elle appelle le «savoir dominant», imposé par le «système dominant». Shiva analyse les transformations 
qui ont affecté la sylviculture et l'agriculture, de leurs formes traditionnelles vers des productions destinées à la vente sur le marché. En Inde, et en général dans les pays considérés comme moins «développés», le changement, à commencer par la «Révolution verte» et jusqu'aux plus récentes applications des biotechnologies, a eu le soutien de la Banque Mondiale et d'autres organisations internationales. Des espèces et des variétés nouvelles, importées de l'extérieur, ont été employées de préférence comme monocultures. Leur productivité, observe Shiva, n'est pas nécessairement plus élevée, et même dans ce cas le calcul est faussé, parce ce qu'on «externalise» les coûts à plus long terme, ceux qui dérivent des effets sur le climat, le système hydrologique, la fertilité du sol. Et surtout, beaucoup de gens ne trouvent plus de possibilité de subsistance sur le territoire et doivent s'en aller.

Avec l'expression «monocultures de l'esprit», Shiva (1993) met en rapport la disparition de variétés végétales et animales et de techniques productives avec celle des alternatives socio-culturelles. La complexité de l'écosystème est détruite et les connaissances traditionnelles, qui permettaient d'en bénéficier en la reproduisant, sont dévaluées. Quand la biodiversité et les savoirs traditionnels ne disparaissent pas, c'est parce qu'ils sont «valorisés», à coups de brevets et en étant soustraits aux populations locales. Celles-ci, en général, perdent le contrôle de leurs conditions d'existence: des ressources comme des connaissances. La perte locale de l'autogouvernement et donc de liberté signale un principe général et un problème global: la réduction de variété et d'alternatives - d'information implique une réduction de liberté.

\section{CE QUI RESTE DU LIBÉRALISME}

F. Hayek, dans le discours prononcé à l'occasion de la fondation de la «Mont Pelerin Society» (MPS) en avril 1947, souligne l'importance de l'opinion publique. Afin de garantir dans le futur un «ordre concurrentiel» réel et efficace, écrit-il, il faut s'employer à diffuser les convictions qui le rendront enfin possible. C'est sur cette base que l'on pourra bâtir un «cadre juridique » convenable et une politique délibérément orientée en faveur «de la concurrence, du marché et des prix » (Hayek 1948: p. 112 et 110).

La description de «l'ordre concurrentiel» par Hayek relève de la plus noble tradition libérale. La propriété privée et la libre entreprise doivent, à son avis, avoir des limites et contribuer à l'avènement de cet ordre. Non seulement les entrepreneurs eux-mêmes doivent respecter les principes concurrentiels avant de l'exiger des travailleurs, mais une opposition de leur part à toute forme d'activité de l'Etat, aussi bien qu'à l'organisation syndicale en tant que telle, serait une erreur. Un impôt sur les héritages pourrait favoriser la mobilité sociale. En revanche, toute loi qui constituerait un point d'appui pour la croissance de monopoles doit être évitée; par exemple, selon Hayek, des «privilèges dangereux » ont été créés «en étendant imprudemment le concept de propriété» à des droits tels que «les brevets d'invention, les droits d'auteur, les marques de fabrique» (p. 113-114).

Hayek présumait qu'un changement aurait pu se réaliser en l'espace d'une génération, et qu'un «ordre concurrentiel» serait alors établi. Sa prévision s'est 
révélée correcte en ce qui concerne le changement opéré dans l'opinion, qu'on pourrait génériquement définir comme «favorable au marché» et qui a pris pied aussi bien dans les milieux scientifiques et politiques que parmi le public. On a même parlé, à ce propos, d'une «pensée unique» qui s'est répandue et imposée (Ramonet 1995). Hayek a apporté une contribution importante en ce sens, non seulement par ses écrits, mais aussi par son initiative de la MPS, dont la mission statutaire est de contrecarrer le «déclin de la foi dans la propriété privée et le marché concurrentiel» et de préconiser «la redéfinition des fonctions de l'Etat» (Mont Pelerin Society 1947).

Depuis 1947, plusieurs autres «think-tanks » ont contribué à ce changement, avec une efficacité incontestable, en ayant recours aux techniques les plus modernes de «Public Relations » et, avant tout, en bénéficiant de financements considérables (voir p. ex. Smith 1991, Desai 1994, George 1996). Le «Institute of Economic Affairs » (IEA), par exemple, créé en 1955 comme branche anglaise de la MPS, a favorisé le tournant politique représenté par le gouvernement Thatcher. Des liens similaires ont été observés aux Etats-Unis, entre cercles économiques conservateurs, institutions culturelles et scientifiques, et la politique du pays, de $\mathrm{R}$. Reagan à l'actuelle hégémonie «neocon». Mais bien avant déjà, la politique économique au Chili, sous la présidence de A. Pinochet, avait été inspirée par l'Ecole de Chicago et en particulier par M. Friedman, qui fut l'un des trente-neuf invités de la réunion du Mont-Pèlerin en 1947.

Il est donc vrai que «les idées ont des conséquences» (Weaver 1948). Dans le cas de Hayek, toutefois, cette affirmation a aussi un sens ironique, car la société néo-libérale des dernières décennies est tout à fait différente de «l'ordre concurrentiel » qui lui tenait à cœur.

Les faits sont bien connus. A partir de la fin des années 1970, une tendance fondamentale du nouvel ordre a été, dans le monde entier, la «dérégulation» des marchés financiers et du travail. En réalité, de nouvelles règles ont été introduites, afin de laisser le champ libre à l'exploitation de tout ce qui - dans la nature, la société et les êtres humains - pouvait être considéré par le système économique comme une ressource «externe». Ce qui a été institué avec succès n'est pas un «ordre concurrentiel», mais au contraire l'accroissement du pouvoir économique et politique des grandes entreprises, la domination du business financier et la destruction des limites législatives posées à la concentration du capital et à l'investissement spéculatif.

Le résultat est que l'apport des salaires au revenu national n'a cessé de diminuer depuis la deuxième moitié des années 1970, que le chômage est devenu structurel, que les fonds publics pour la santé et la sécurité sociale ont été réduits, qu'une partie croissante des travailleurs ont été forcés à accepter des emplois précaires, sous-qualifiés et mal payés. Les inégalités ont augmenté, aussi bien entre les différentes couches sociales qu'entre les pays «développés» et les autres. Dans ces derniers, selon les estimations de la FAO, vivent la plupart des 852 millions de personnes qui souffrent de la faim ainsi que la plupart des six millions d'enfants qui meurent chaque année de dénutrition (FAO 2005).

Les coûts sociaux et les dégâts écologiques provoqués par le développement capitaliste dans un monde de plus en plus unifié sont souvent dissimulés ou minimisés, et la raison de cette attitude est aussi celle qui les a générés: ils sont perçus par le système économique comme «extérieurs», et cela explique pourquoi le 
système social a tant de difficulté à en tenir compte - à les faire passer de «brouillage informatif» en «information». Quand les problèmes ne sont pas ignorés, les remèdes proposés peuvent être illusoires et même contre-productifs. Le problème de la faim est bien documenté dans le réseau mondial de l'information: mais dans quelle mesure peut-il trouver une solution à l'intérieur du système qui le produit, où l'information proprement dite, celle qui compte, est celle de la «monoculture de l'esprit»?

En ce qui concerne le système politique, la crise des institutions démocratiques, qui a accompagné les transformations des institutions économiques, apporte un démenti à la confiance qu'avait Hayek dans la liberté économique comme garantie de la liberté tout court. La formulation synthétique de K. Polanyi, dans les pages conclusives de La grande transformation, semble encore plus appropriée aujourd'hui qu'il y a soixante ans: «Dans la pensée libérale l'idée de liberté $[. .$. ] dégénère en simple soutien de la libre entreprise - qui actuellement est réduite à une fiction par la dure réalité des trusts géants et du pouvoir des monopoles» (Polanyi 1957: p. 257). Le problème de la liberté est immédiatement lié, comme cela est typique de la pensée de Polanyi, à celui de la démocratie: en effet, poursuit-il, « la plénitude de la liberté » n'est accessible qu'à une minorité pourvue de propriété et de pouvoir tandis qu'il ne reste qu' «une misère de liberté pour la plupart des gens, qui cherchent, souvent en vain, à user de leurs droits démocratiques » (ibid.).

C. Crouch (2003: p. 6-7) appelle «post-démocratie» le modèle «minimaliste» actuel de démocratie. A son avis, les sociétés capitalistes ont renoncé à l'idée d'une démocratie «réelle», dans laquelle une véritable information devrait permettre une participation responsable des individus et de leurs associations à la définition des choix publics. Les décisions politiques sont prises à l'intérieur de l'interaction entre le personnel politique et des élites, qui représentent surtout des intérêts économiques. La sauvegarde de l'ordre politique, c'est-à-dire des rapports de pouvoir existants, devient la préoccupation principale des Etats et des institutions internationales.

Selon Crouch, à partir de Reagan le concept de démocratie en Amérique tend à se réduire à l'existence d'élections libres. Il faut ajouter que le droit de vote est formellement garanti à tout le monde, mais pratiqué par une minorité de citoyens, et que le choix des électeurs est très limité. On soupçonne même que, au cours des récentes élections, leur choix n'ait pas été respecté. Enfin, la domination exercée sur l'opinion publique par les moyens de communication de masse, et donc par les grandes firmes qui les possèdent ou les financent avec la publicité, nous amène à réfléchir avec une préoccupation croissante à l'observation de J. Schumpeter, à savoir que l'activité visant à former l'opinion des électeurs et à orienter leur choix «est un aspect essentiel du processus démocratique » (Schumpeter 1943: p. 282).

La démocratie «formelle» restante est elle-même menacée, non seulement à cause de situations d'urgence qui deviennent «guerre permanente», mais en général à cause de l'absence d'une participation plus large, informée et responsable des citoyens à la vie politique. On peut aussi rappeler à ce propos le cercle vicieux signalé par Crouch: à mesure que la politique se retire des problèmes concernant la vie des gens, ceux-ci deviennent plus indifférents envers la politique, et les grandes compagnies multinationales sont alors en condition de les 
exploiter plus aisément, en contrôlant l'agenda politique et en manipulant l'opinion publique (Crouch 2003: p. 25-26).

En conclusion, «l'ordre concurrentiel» préconisé par Hayek est resté une utopie: mais cela n'a pas empêché ses idées, et celles d'autres chercheurs libéraux, de fonctionner comme un support pour l'évolution historique des dernières décennies. Leur efficacité semble reposer justement sur l'image illusoire de la société de marché qu'elles diffusent.

Peu de temps avant le congrès réuni au Mont-Pèlerin, Polanyi publia son article sur «notre obsolète mentalité de marché». Obsolète certes, mais encore utile dans la lutte politique entre deux tendances opposées. La première tendance, écrit Polanyi dans la conclusion de son article (Polanyi 1947: p. 117), vise «à une société authentiquement démocratique», dans laquelle l'économie serait organisée «à travers l'intervention planificatrice des producteurs et des consommateurs eux-mêmes. Cette activité consciente et responsable est effectivement un moyen pour réaliser la liberté dans une société complexe». La deuxième tendance, opposée à la première, conduit vers une société «adaptée plus profondément au système économique», dont les caractéristiques essentielles restent inchangées. Les tenants de cette tendance sont les gens qui «croient dans les élites et les aristocraties, dans le managérialisme et la grande entreprise», et les gens du commun qui sont «conditionnés » à y croire.

Entre les deux tendances, il n'y a pas de place pour «l'ordre concurrentiel»; Polanyi considère en effet périmée - emportée par la crise irréversible qui l'a touchée et par la «grande transformation»- la «structure institutionnelle» de la société libérale. Cela n'empêche pas qu'une «obsolète mentalité de marché» puisse fonctionner comme une arme idéologique en faveur de l'une des deux tendances de la société post-libérale, et il n'est pas nécessaire de préciser laquelle.

\section{LA CRITIQUE INSTITUTIONNALISTE}

Hayek reformule brillamment la théorie de l'équilibre économique en termes de «connaissance»; il prétend ainsi dépasser le modèle formel et tautologique de l'équilibre et transformer la science économique. Celle-ci cesserait d'être «un exercice de logique pure» pour devenir une «science empirique» (Hayek 1937: p. 44). Au lieu de l'équilibre comme résultat, comme modèle abstrait, Hayek considère les combinaisons, toujours provisoires et continuellement renouvelées, des choix et donc des plans des individus. La connaissance, dont ces plans dépendent, est incomplète et orientée vers des objets particuliers, parce que chaque individu connaît uniquement les choses qui lui sont plus proches et qui ont quelque importance pour ses intérêts («relevant knowledge»). Le «système des prix», tout en réalisant l'adaptation réciproque des plans des individus, fonctionne comme «un mécanisme pour communiquer de l'information» (Hayek 1948: p. 86). Ce système, en effet, se fonde sur la concurrence, considérée comme un processus qui fait circuler l'information et réalise de cette façon, mieux qu'aucune autre méthode, «l'unité et la cohérence du système économique » en même temps que la «satisfaction des consommateurs» (Hayek 1948: p. 105-106).

En dépit de sa contribution originelle à l'analyse du mécanisme du marché, Hayek reste clairement à l'intérieur du paradigme néo-classique (et néo-libéral). 
Selon lui, la connaissance que les individus mettent en œuvre pour opérer leurs choix concerne des données, à propos desquelles aucun problème n'est soulevé; elles sont simplement le résultat des choix de tous les individus, qui se présente à chacun d'eux, justement, comme «donné». C'est une perspective individualiste, qui fait abstraction du système social et évite par conséquent tout problème concernant deux dynamiques fondamentales: la sélection de l'information opérant au niveau du système et la «causalité cumulative» qui, d'après T. Veblen (1898), est inhérente à la vie économique considérée comme processus historique, «évolutif». Ces dynamiques - qui relèvent de l'organisation de la production et de l'échange, et ont toujours été centrales dans les théories institutionnalistes influencent les motifs des choix individuels et en déterminent les conditions. Elles deviennent visibles seulement quand le marché n'est pas considéré comme le lieu idéal des activités et des échanges en général, mais comme une institution économique spécifique, qui se développe historiquement comme processus de circulation de l'économie capitaliste. La structure du marché comme les données des choix changent cumulativement et, en général, irréversiblement. Le contexte des choix individuels est donc, du point de vue institutionnaliste, la question prioritaire et fondamentale. Les données, les conditions et les contraintes des choix dépendent de l'évolution du système du marché capitaliste qui, comme tous les systèmes, se reproduit en sélectionnant et en utilisant l'information qui lui convient, suivant son organisation.

Ce qui caractérise le paradigme institutionnaliste en économie est l'étude comparative des systèmes économiques, en tant qu'organisations sociales historiquement spécifiques. A. Gruchy (1947) appelle à juste titre «holistic economics » l'économie institutionnaliste, puisque seule l'analyse de la société dans son ensemble - d'une société donnée dans un temps donné - permet de comprendre son organisation économique. Polanyi parle en ce sens du problème de «la place de l'économie dans la société». L'activité économique s'avère ainsi socialement définie et coordonnée: d'une part, l'économie a une «forme»sociale, elle est «institutionnalisée » et, d'autre part, les actes économiques de chacun sont «intégrés », comme le dit Polanyi, dans un système. Polanyi oppose cette conception à celle de L. Robbins, lequel, au contraire, fait abstraction de toute spécificité institutionnelle et considère comme «économique » un certain «aspect» du «comportement humain», on pourrait même dire une certaine attitude: celle qui consiste à mettre rationnellement en rapport des fins données avec des «moyens insuffisants qui ont des usages alternatifs » et qui sont également donnés. Fins et moyens ne sont donc pas objet d'analyse en tant que tels, mais seulement pour le fait que l'insuffisance des moyens impose d'optimiser leur distribution entre leurs emplois possibles. Ce sont justement les conditions de «rareté », précise Robbins, qui imposent la «forme » économique du comportement, quels que soient le temps et le lieu (Robbins 1962: p. 16-17).

L'opposition entre le paradigme institutionnel et celui de la théorie dominante néoclassique est représentée de façon incisive par la signification que l'un et l'autre donnent respectivement au concept de «forme»: organisation sociale d'un côté, type de comportement de l'autre. Le mérite de Polanyi a été de démontrer que cette dernière signification est le résultat d'une généralisation indue des caractères spécifiques de la société de marché et capitaliste. C'est dans cette société que l'activité économique consiste à «économiser» en vue d'un résultat 
quantitatif et monétaire. Le but de la production n'est plus, comme dans les sociétés précédentes, la satisfaction de besoins donnés, grâce à des moyens et un rapport d'équilibre avec l'environnement naturel également donnés par la culture traditionnelle: l'argent - «medium universel», «rapport social» selon K. Marx est, au contraire, le but.

Comme tout le monde le sait, on n'a jamais assez d'argent pour joindre les deux bouts. La rareté, alors, n'est plus seulement une condition générale de l'humanité chassée du Paradis; elle devient aussi un trait spécifique de l'organisation sociale, elle devient systémique, dans le sens qu'elle est inhérente au fonctionnement du système économique lui-même. Cette connotation, que la forme sociale donne à la rareté, est constitutive, avec les autres aspects fondamentaux du système de marché, du «contexte informatif » (Wilden 1978: p. 606) dans lequel se déroule la vie des individus. C'est en ce sens que la rareté fait partie des habitudes, des désirs et des valeurs codifiés. Et on la retrouve en ce sens à la base des motifs de l'activité économique dans «les conditions d'une économie de marché », dans lesquelles, selon M. Weber, «l'impulsion décisive» est représentée par «l'acquisition rationnelle» en vue du profit d'un côté, et, de l'autre, par «le risque d'un manque total d'approvisionnement» (Weber 1980: p. 106). Polanyi reprend ces «motifs » dans sa théorie de la «société de marché» considérée comme société où la sphère économique s'autonomise et constitue, par conséquent, la contrainte fondamentale, et la seule qui soit générale, de l'organisation sociale. Il y a d'abord «le principe du gain et du profit», qui devient «la force organisatrice dans la société» (Polanyi 1957: p. 170); l'autre motif de l'activité économique, la faim, est analysé dans La grande transformation comme élément fonctionnel du système social, comme condition, en particulier, de l'établissement du marché du travail. En comprenant la spécificité historique du gain et de la faim comme motifs «purement économiques », Polanyi est aussi en condition de fonder sa critique de l'économisme comme généralisation erronée des caractères que l'activité économique présente dans une forme particulière de société.

La définition «formelle» de l'économie par Robbins relève de ce type de généralisation. Pour lui (comme pour Hayek) le marché et l'entreprise capitaliste - l'un et l'autre agissant dans un «ordre» qu'on souhaite «concurrentiel» - se présentent comme les sujets naturellement chargés de la fonction d'opérer des choix rationnellement économiques ou, plus précisément, «économisants ». Les fins sont données parce qu'on suppose qu'elles sont déterminées ailleurs: par les individus, les mœurs, la politique. Les ressources (y compris le travail humain) et la technique étant également donnés, voilà que le système économique se présente comme ce qu'il devrait être, un simple moyen pour satisfaire - rationnellement les besoins. Et le marché apparaît à son tour comme le système le plus efficace pour remplir cette fonction.

Robbins cite Weber pour soutenir sa vision «formelle» de l'économie et considérer les fins et les valeurs comme donnés. Il est vrai que Weber, après avoir défini la «rationalité formelle» de l'économie - qui se réalise pleinement avec le «calcul du capital» dans la «situation de marché»- lui oppose la «rationalité matérielle», qui caractérise l'économie comme moyen pour des fins et des «postulats de valeur», établis - en principe - dans d'autres sphères de la vie individuelle ou sociale (Weber 1980: p. 80-81). Mais pour Weber il s'agit d'une 
distinction conceptuelle, alors que Robbins suppose qu'elle est concrètement réalisée dans la société. Cette divergence est fondamentale pour comprendre l'opposition entre les deux paradigmes, néoclassique et institutionnaliste.

Le fait que, selon Weber, «la rationalité formellement plus parfaite du calcul du capital» soit «indifférente» à quelque «postulat matériel» que ce soit, implique la possibilité que ce qui devrait rester «formel» ait une influence «matérielle». La «rentabilité », qui est «une catégorie formellement rationnelle», peut déterminer, observe en effet Weber, «le choix des besoins à satisfaire» (ibid.: p. 90). La concurrence, le marché, sont le théâtre d'une «lutte», aboutissant à des différences de pouvoir d'achat, et de pouvoir tout court, qui sont « décisives pour déterminer la production des biens » (ibid.: p. 88-90). Les entrepreneurs ne prennent en compte les besoins que du point de vue du pouvoir d'achat qui les accompagne et orientent leur choix sur ceux qui leur assurent la plus grande «rentabilité». Ils arrivent même à «susciter» et «influencer» les besoins au moyen d'une «publicité agressive» (ibid.: p. 95).

Ces affirmations de Weber, comme l'aperçu minimal d'idées institutionnalistes qui va suivre, semblent suggérer une signification que la «marchandisation de l'information» peut aussi avoir: l'information qui conditionne l'activité économique reste délimitée dans le cadre institutionnel du marché, de la valorisation monétaire, de la «rationalité formelle». En adoptant la définition plus générale, que Polanyi appelle «substantive», de l'économie comme activité sociale qui pourvoit aux besoins des hommes à travers l'interaction avec la nature, on sort de la rationalité marchande. On ouvre ainsi, en effet, un domaine informatif plus vaste, on rend possible une plus grande variété d'informations.

Bien avant la fin du $\mathrm{XIX}^{\mathrm{e}}$ siècle, la divergence entre la théorie de l'équilibre et la réalité historique des institutions économiques était devenue évidente. Weber est ainsi amené à mettre en question les postulats de la théorie économique, comme nous venons de le voir. Même l'Ecole Autrichienne - qui est la source principale des conceptions économiques de Weber - semble, à ses débuts, prendre acte de cette divergence, avec la distinction, qu'on trouve chez $\mathrm{C}$. Menger et F. Wieser, entre la «valeur naturelle» ou «subjective» d'un côté et, de l'autre, la «valeur objective» correspondant au prix (Wieser 1889). Les biens seraient échangés à leur valeur naturelle - et, par l'intermédiaire des valeurs, la consommation et la production correspondraient le mieux possible aux besoins de tous les individus composant la société - seulement dans une situation idéale où la rationalité de chacun et l'information dont il bénéficie seraient parfaites, tout comme l'élasticité des prix. Ces derniers, en outre, ne seraient en aucune manière influencés par l'organisation sociale. Par contre, selon Wieser, dans la réalité les institutions sociales, à commencer par la propriété privée, provoquent une distribution systématiquement inégale du pouvoir d'achat et, par conséquent, une déviation des prix par rapport aux valeurs naturelles. Menger à son tour affirme que «dans la société actuelle, fondée sur l'échange» les entrepreneurs sont intéressés par les besoins des membres de la société seulement si, et dans la mesure où, ces besoins sont accompagnés de «la capacité et la volonté de payer » (Menger 1923, p. 50).

Cette question restera d'actualité. On la retrouve par exemple dans «l'économie du welfare» de C. Pigou et surtout elle se présente comme une évidence incontournable chez les économistes institutionnalistes. L'Ecole Autrichienne, 
dans ses développements ultérieurs, aura au contraire tendance à l'oublier, de même que toute référence à la réalité institutionnelle. Cette Ecole, et la théorie néoclassique en général, prendront une direction que Schumpeter désigne sous le nom d' «analyse économique», par opposition à «l'économie politique »: une théorie de plus en plus abstraite, pure «boîte à outils» apte à analyser n'importe quelle réalité économique.

A l'époque de la théorie classique, le raccourci utilitariste permettait de tenir pour acquis que l'aisance et le bonheur des individus et de la société résultaient du fonctionnement de la sphère économique, et qu'il fallait donc la laisser se déployer suivant ses propres règles. Si cette vision trouvait quelque appui dans la réalité du capitalisme à ses débuts, réplique Veblen, elle est démentie par le développement même du capitalisme. Il devient de plus en plus clair que le profit n'est pas un moyen visant à des finalités sociales, mais une fin en soi. La question soulevée par Wieser et Menger se trouve radicalisée dans la distinction tracée par Veblen (p. ex. 1901: p. 309) entre «vendibility» et «profitability» d'une part et «serviceability» de l'autre; c'est-à-dire, entre ce qui convient aux producteurs capitalistes et ce qui est utile pour la société. Dans le même article (p. 286), l'activité «pécuniaire», celle qui vise au gain monétaire, est présentée comme le facteur décisif («controlling factor») du processus économique moderne. Dans la première page de sa Theory of Business Enterprise, Veblen n'hésite pas à indiquer le profit comme «force directrice» du «système capitaliste», en particulier quand on passe des petites entreprises au «big business». Ce qui peut être intéressant dans certaines expressions employées par Veblen est qu'elles rappellent des concepts bien plus récents de la théorie des systèmes, tels que «fonction de contrôle» et «téléonomie».

Un point de vue institutionnaliste, en continuité avec Veblen et en opposition avec les propositions méthodologiques de Robbins, est développé dans Economics and Sociology, le livre publié en 1935 par A. Löwe. L'analyse de la «transformation évolutive» du système économique, écrit Löwe (1935: p. 138), implique celle des changements correspondants de la «constellation sociologique». Et inversement, ces derniers sont liés aux développements techniques et institutionnels de l'économie. Par exemple, «l'optimisme social» des économistes classiques quant aux bienfaits du marché et au motif du profit présupposait une production à forte intensité de travail; la mobilité technique et sociale pouvait alors être la base de la loi de l'offre et de la demande comme celle du développement; de l'efficacité économique comme de la justice sociale. La concentration du capital et les investissements à forte intensité de capital s'accompagnent de conditions techniques, économiques et sociales bien différentes. Löwe parle par exemple, à ce propos, non seulement de la diminution de l'élasticité de l'offre et du ralentissement des investissements dus à la masse croissante des amortissements, mais aussi de la formation de monopoles et de l'impérialisme, en tant que phénomènes significatifs dans le rapport qu'ils entretiennent l'un avec l'autre et à l'intérieur du processus évolutif d'un système économique et social spécifique (ibid.: p. 109-110).

L'économie «pure» à la Robbins porte à supposer, écrit Löwe, que «les conditions naturelles, sociales et techniques de la production ont leur origine exclusivement hors de la sphère économique» (ibid.: p. 105). Mais la réalité est tout autre. D'un côté, « l'appropriation privée des facteurs de la production est déterminante 
pour l'ordre de la production»; de l'autre, c'est le processus économique luimême qui «produit et change ses propres données» (ibid.: p. 108 et 97). Ce processus est influencé par le milieu politique et social, mais il l'influence à son tour; le progrès technique et l'investissement, en effet, «ont donné à ceux qui possèdent le capital ou en disposent la suprématie non seulement économique, mais aussi sociale et politique » (ibid.: p. 113).

R. Tawney, en 1920, souligne l'expression «féodalisme industriel», employée dans le «Final Report of the United States Commission of Industrial Relations » de 1916. Il parle à ce sujet de la «concentration de pouvoir» qui, avec le développement des grandes entreprises, déborde de la sphère des relations industrielles pour déterminer la vie entière des individus: non seulement leur subsistance matérielle, mais aussi leur existence sociale et les choix politiques qui les concernent (Tawney 1920: p. 129). Les études sur ces thèmes sont nombreuses; je me limite à rappeler, à titre d'exemple, celles de C. Wright Mills sur «l'élite du pouvoir» et celles de A. Berle. Ce dernier, en 1954, traite encore des grandes entreprises comme d' «institutions qui organisent le pouvoir» (Berle 1954: p. 22) non seulement à l'intérieur de leur propre structure, mais aussi à l'intérieur de la société et du rapport qu'elle entretient avec son environnement.

Ces problèmes n'ont pas disparu avec la fin du «fordisme » et l'avènement du néolibéralisme et de la «mondialisation»: au contraire. Plusieurs ouvrages continuent à aborder ces sujets de nos jours. Mais on a aussi l'impression que les sciences sociales académiques, et l'économie en particulier, mettent tout en œuvre pour laisser de côté ces problèmes, et même pour sélectionner les instruments théoriques les plus aptes à les garder hors de l'horizon cognitif. En particulier, une conception réductrice de l'information en économie, qui présuppose, comme nous l'avons vu, une définition «formelle» de l'économie, ne peut qu'aboutir à une carence d'information ou à une information trompeuse sur la réalité sociale et ses problèmes.

La connaissance dont parle Hayek reste enfermée à l'intérieur du système économique car il s'agit d'une connaissance utile aux individus en tant que producteurs et consommateurs, dans le système de marché. La perspective ne change pas beaucoup, par exemple, quand K. Arrow (1974) met le problème de l'information en rapport avec celui de l'efficacité avec laquelle les organisations poursuivent leur but. Les organisations évoquées sont les entreprises capitalistes et le but est le profit, mais cela reste implicite, quoique ce soit précisément de cela que la conclusion et même le problème de Arrow semblent dépendre: la valeur de la concentration du pouvoir décisionnel dérive du fait que l'information a un coût, qui augmente en proportion de la nécessité de la partager. La conclusion, et le problème, resteraient-ils les mêmes si le but était différent ou s'il s'agissait de choisir le(s) but(s)?

Les résultats de la science économique «normale» sont souvent brillants et utiles, comme dans le cas de Arrow: mais ils le sont à l'intérieur du système de marché, et donc à condition qu'ils ne prétendent pas avoir une signification et une utilité plus générales. Par exemple, à condition qu'ils ne prennent pas la place d'une analyse de la réalité du processus économique et social et de ses transformations institutionnelles.

Ce qui caractérise, par contre, le paradigme institutionnaliste est de se situer hors du système: du point de vue de la méthode, en considérant le système comme 
tel, c'est-à-dire comme un élément de l'ensemble de tous les systèmes économiques; du point de vue théorique, en considérant comme centraux les deux problèmes, liés l'un à l'autre, de l'évolution historique du système et de la communication du système avec son environnement. Un seul exemple à ce propos: K. W. Kapp - une exception relativement récente au ralliement tendanciel des économistes à la normalité néoclassique - demande explicitement de ne pas considérer l'économie comme un système fermé, et déplace le centre de la problématique économique à la frontière entre le système et son environnement. Le flux d'information qui passe - ou ne passe pas - par cette frontière devient alors la question principale. Les «coûts sociaux » dont parle Kapp (1963) ne sont pas mis en compte dans les bilans des entreprises mais, bien qu'ils soient inhérents à leur activité, sont reportés sur l'environnement: sur les individus, sur le fonctionnement de la vie sociale, sur la nature. Ils sont une «information»-une «différence qui fait une différence», comme le dit G. Bateson - dans d'autres systèmes, qui constituent l'environnement du système économique; mais celui-ci ne traite pas cette information, car il ne la reconnaît même pas.

Il y a là une « dissymétrie d'information » et une « rationalité limitée » bien différentes de celles qui intéressent les économistes, y compris les néo-institutionnalistes, et qui concernent les transactions de marché entre individus ou entreprises. La mise en question des «coûts sociaux» dans le sens de Kapp soulève le problème plus général de la fermeture du système économique. Quand le mécanisme du marché et la contrainte du profit s'imposent dans l'activité économique, le système économique tend à devenir autoréférentiel. Il tend alors à avoir une attitude instrumentale par rapport à tout ce qui est «extérieur», à ignorer les contraintes concernant son environnement naturel, social et humain, aussi bien que les conséquences de son fonctionnement sur l'environnement. Autrement dit, il tend à sélectionner les informations en fonction de la reproduction de sa propre organisation.

Une information aussi réductive a un effet désorganisant, entropique, sur le système social et l'écosystème terrestre dans lesquels le système économique est inséré et desquels il dépend pour sa survie. J. O’Connor (1991) considère cette contradiction comme prééminente dans le capitalisme contemporain. La croissance économique n'a été possible, à son avis, qu'au prix du pillage et de la détérioration de l'environnement humain, social et naturel, tout en ignorant ou en dissimulant ces coûts, vu qu'ils pouvaient être « externalisés »: mais cela entraîne, à la longue, une augmentation des coûts dans le système économique lui-même. Comme celui-ci ne peut donc se soustraire à l'augmentation du désordre qu'il provoque, il tend à en provoquer davantage, puisqu'il cherche à déplacer sur l'environnement les coûts ultérieurs aussi. Le processus, le cercle vicieux, peut finir par échapper à tout contrôle.

\section{UNE LIBERTÉ CYBERNÉTIQUE}

Le problème de la politique, qui se pose alors, concerne la prédominance des contraintes économiques dans la société de marché. La liberté des choix sociaux s'avère lourdement limitée par ces contraintes. La politique ne devrait-elle pas renverser ce rapport? Comment la société, par ses institutions politiques, pourraitelle définir les fins et les contraintes de l'activité économique? 
Kapp résume et affine ses réflexions méthodologiques dans son dernier article, dont le titre - «The Nature and Significance of Institutional Economics » - est une paraphrase de celui de l'Essay de Robbins. Dans la conclusion de son article, il se rallie à «la nouvelle approche normative aux sciences sociales et à l'action sociale» (Kapp 1976: p. 228). Au début, il cite R. Lynd: les sciences sociales doivent être capables d'analyser la société dans son ensemble et de soulever, en s'appuyant sur cette base, la question de savoir «quels types d'ordre, d'organisation, peuvent être créés, qui soient plus utiles » à la société et à ses membres (Lynd 1939: p. 126). L'approche de ceux qui, en confiant le destin de l'humanité à la rationalité économique et à la loi des prix de marché, évitent le problème du rapport du système économique avec la société, et de la société dans son ensemble avec son environnement, est aussi une approche normative. La différence est qu'ils ignorent le problème, en tenant pour acquis que l'ordre existant, la norme dominante sont la solution naturelle. Trop souvent la science économique appuie son «objectivité » sur une conception réductrice du problème économique et ses découvertes sur l'occultation d'informations cruciales.

La perspective totalement différente adoptée par Polanyi le porte à mettre en question «la productivité sociale de l'économie capitaliste». C'est une économie qui ne peut tenir compte ni des évaluations sociales ni des «effets en retour du processus de production sur la vie de la communauté»; qui manque d'un «organe de perception » pour considérer «comment le bien-être général se voit favorisé ou détérioré par telle ou telle orientation de la production ou mode de la production » (Polanyi 1922: p. 389-390).

La théorie politique que Polanyi cherche à définir dans ces années-là se base sur l'idée que l'efficience des organisations, leur capacité de répondre au mieux aux besoins individuels et sociaux, dépend de la quantité d'information qu'elles arrivent à prendre en considération et à utiliser. Le côté «autrichien» de Polanyi, et même ce qu'il peut avoir en commun avec Hayek, apparaît dans sa préoccupation d'impliquer dans le processus informatif-politique tous les sujets, en tant que porteurs de connaissances particulières dont la combinaison permettra d'avoir la meilleure «vision» générale, et donc la meilleure politique. La différence, voire l'opposition avec Hayek est pourtant évidente: le rôle principal, dans la fonction sociale de susciter, diffuser et coordonner l'information, ne doit pas être joué, selon Polanyi, par le marché et les prix, mais par un processus politique, le plus possible conscient, répandu et démocratique. Démocratie, information et efficience sont liées et directement proportionnelles. «La capacité qu'ont les organisations - écrit Polanyi - d'accomplir leur fonction dépend de la quantité de démocratie active présente dans la vie quotidienne »; et cela est vrai aussi bien pour une usine que pour un parti politique ou un Etat (Polanyi 1925: p. 23-24).

Une société capable de résoudre les problèmes et les conflits, d'évaluer les «effets en retour» de son fonctionnement, et d'augmenter ainsi les possibilités et donc la liberté des sujets, est une société ouverte, où les contraintes systématiques inhérentes à sa propre organisation sont réduites le plus possible. Liberté et démocratie vont du même pas. Polanyi donne à ces deux termes une signification «substantielle» et «positive», qui porte sur la conscience et la responsabilité des individus vis-à-vis des choix et des contraintes sociales. L'utilitarisme, à son avis, est illusoire dans la mesure où le marché est plutôt un «mécanisme» dont les individus dépendent que le règne de la liberté; et aussi pour une raison concernant la 
condition générale de l'homme, qui peut être libre seulement dans la société, dans et à travers des normes, à la formulation desquelles, dans la société moderne, il est appelé à prendre consciemment part.

La crise de la société libérale et de la confiance dans l'autorégulation de l'économie de marché amène aussi $\mathrm{K}$. Mannheim au problème de la régulation consciente de la société et, en premier lieu, de l'économie. La réorganisation nécessaire de la société industrielle, l'intégration et la collaboration qu'il faut créer dans la société lui semblent des tâches aussi inévitables que difficiles, qui exigent un grand effort de compréhension, de «rationalité substantielle». La nature complexe et dynamique de la société moderne demande que cette rationalité, définie comme la capacité d'agir avec intelligence dans des situations concrètes, soit répandue et cultivée le plus possible. Mais, observe Mannheim (voir 1954: chap. II), l'organisation de la société industrielle présuppose la concentration des moyens de production comme de la connaissance, et par conséquent celle du pouvoir de faire des choix.

\section{Dipartimento di Studi Storici \\ Università «Ca' Foscari» di Venezia \\ cangiani@unive.it}

\section{RÉFÉRENCES BIBLIOGRAPHIQUES}

Arrow K., The Limits of Organization, New York, Norton \& Co., 1974.

Azam G., «L'utopie de l'économie de la connaissance », Sciences de la société, n 66, 2005, p. 15-28.

Berle A. A., The $20^{t h}$ Century Capitalist Revolution, New York, Harcourt, Brace \& Co., 1954.

Coriat B., «Le nouveau régime américain de la propriété intellectuelle», Revue d'économie industrielle, $\mathrm{n}^{\circ}$ 99, 2002, p. 17-32.

Crouch C., Postdemocrazia, Bari, Laterza, 2003 (trad. ital., augmentée, de Coping with Post-democracy, London, The Fabian Society, 2000).

Desai R., «Second-Hand Dealers in Ideas: Think-Tanks and the Thatcherite Hegemony », New Left Review, n 203, 1994, p. 27-64.

FAO, L'état de l'insécurité alimentaire dans le monde, Roma, 2005.

George S., «Fonds et Fondations. Comment la pensée devient unique», Le Monde Diplomatique, septembre 1996.

Gruchy A. G., Modern Economic Thought. The American Contribution, New York, Prentice-Hall, 1947.

Hayek F. A., «Economics and Knowledge», Economica, IV, 1937, p. 33-54.

- $\quad$ "The Use of Knowledge in Society », in Individualism and Economic Order, Chicago, University of Chicago Press, 1948.

Kapp K. W., The Social Costs of Business Enterprise, Nottingham, Spokesman, 1963.

- $\quad$ "The Nature and Significance of Institutional Economics », Kyklos, XXIX, n² 2, 1976, p. 209-232.

Löwe A., Economics and Sociology, London, George Allen \& Unwin, 1935.

Lynd R. S., Knowledge for What? The Place of Social Science in American Culture, New York, Grove Press, Inc., 1964 (orig. publ. Princeton, Princeton University Press, 1939).

Mannheim K., Man and Society in an Age of Reconstruction, London, Routledge \& Kegan Paul, 1954 $\left(1^{\mathrm{e}}\right.$ version allemande 1935$)$ 
Menger C., Grundsätze der Volkswirtschaftslehre, publié par K. Menger, Vienna \& Leipzig, HölderPichler-Tempsky \& G. Freytag, 1923.

Mont Pelerin Society, «Statement of Aims», 1947, <www.montpelerin.org>.

O'Connor J., «The Second Contradiction of Capitalism: Causes and Consequences», Capitalism, Nature, Socialism, $\mathrm{n}^{\circ}$ 8, 1991.

Polanyi K., «Sozialistische Rechnungslegung», Archiv für Sozialwissenschaft und Sozialpolitik, IL, 1922, p. 377-418.

- «Neue Erwägungen zu unserer Theorie und Praxis», Der Kampf, XVIII, n 1, 1925, p. 18-24.

_ «Our Obsolete Market Mentality», Commentary, III, n², 1947, p. 109-117.

- $\quad$ The Great Transformation, Boston, Beacon Press, 1957.

Ramonet I., «La pensée unique», Le Monde Diplomatique, janvier 1995.

Robbins L., An Essay on the Nature \& Significance of Economic Science, London, Macmillan, 1962 (1 ${ }^{\mathrm{e}}$ éd. 1932).

Shiva V., Monocultures of the Mind. Perspectives on Biodiversity and Biotechnology, London, Zed Books, 1993.

Smith J. A., The Idea Brokers: Think-Tanks and the Rise of the New Policy Elites, New York, The Free Press, 1991.

Schumpeter J. A., Capitalism, Socialism, and Democracy, London, Georg Allen \& Unwin, 1943.

Tawney R., The Acquisitive Society, New York, Harcourt, Brace \& Co., 1920.

Veblen T., «Why is Economics not an Evolutionary Science?» (1898), in The Place of Science in Modern Civilization, London, Routledge/Thoemmes Press, 1994.

- «Industrial and Pecuniary Employments» (1901), in The Place of Science in Modern Civilization, London, Routledge/Thoemmes Press, 1994.

- $\quad$ The Theory of Business Enterprise, New York, Ch. Scribner's Sons, 1904.

Weaver R., Ideas Have Consequences, Chicago, University of Chicago Press, 1948.

Weber M., Economia e società, vol. 1, Milano, Edizioni di Comunità, 1980.

Wieser F., Natural Value, New York, Kelley \& Millman, 1956 (Der natürliche Wert, 1889).

Wilden A., «Comunicazione», in Enciclopedia, vol. 3, Torino, Einaudi, 1978. 